\title{
Regiocontrolled Synthesis of Pyrrole-2-carboxaldehydes and 3-Pyrrolin-2-ones from Pyrrole Weinreb Amides
}

\author{
Aaron R. Coffin, Michael A. Roussell, Elina Tserlin, and Erin T. Pelkey* \\ 300 Pulteney St., Department of Chemistry, \\ Hobart and William Smith Colleges, Geneva, NY 14456 \\ pelkey@hws.edu
}

\section{Supporting Information Table of Contents}

$\begin{array}{ll}\text { (i) General Methods } & \text { S2 }\end{array}$

$\begin{array}{ll}\text { (ii) } & \text { Experimental Procedures and Spectral Data (5-7) }\end{array}$

(iii) Spectral Data for Pyrrole Weinreb Amides 1a-1d $\quad$ S4

$\begin{array}{ll}\text { (iv) Spectral Data for Pyrrole-2-carboxaldehydes 2a-2d } & \text { S7 }\end{array}$

(v) Spectral Data for 3-Pyrrolin-2-ones 3a-3d (1H-pyrrol-2(5H)-ones) S9

(vi) References $\quad$ S11 


\section{(i) General Methods}

All reactions were performed under a positive argon atmosphere with magnetic stirring unless otherwise noted. Tetrahydrofuran (THF) was distilled from sodium-benzophenone ketyl. Dichloromethane and triethylamine were distilled from calcium hydride. Unless otherwise indicated, all other reagents and solvents were purchased from commercial sources and were used without further purification. ${ }^{1} \mathrm{H}$ NMR and ${ }^{13} \mathrm{C}$ NMR chemical shifts are reported in parts per million $(\delta)$ using the solvents residual proton or carbon signal $\left(\mathrm{CDCl}_{3}: \delta \mathrm{H} 7.24 \mathrm{ppm}, \delta \mathrm{C} 77.3\right.$ ppm; $\mathrm{d}_{6}$-DMSO: $\delta \mathrm{H} 2.50 \mathrm{ppm}, \delta \mathrm{C} 39.5 \mathrm{ppm}$ ) as an internal reference. All yields are for chromatographed or recrystallized, isolated materials.

Known $\beta$-nitroacetates $\mathbf{8 a},{ }^{1} \mathbf{8 b},{ }^{2}$ and $\mathbf{8} \mathbf{c}^{2}$ were prepared using the procedure of Barton and co-workers. ${ }^{2}$ Known $\alpha$-nitroalkenes $9 \mathbf{a}, \mathbf{9 b}$, and 9c were prepared using the procedure of Ballini and co-workers. ${ }^{3}$ Boc-glycine $(\mathbf{4})^{4}$ and 1-nitro-1-cyclohexene $(\mathbf{9 d})^{5}$ are commercially available. $\alpha$-Nitrostilbene $(\mathbf{9 e})^{6}$ was obtained by a Henry reaction between phenylnitromethane ${ }^{7}$ and benzaldehyde in the presence of $n$-butylamine (toluene, reflux, Dean-Stark apparatus).

\section{(ii) Experimental Procedures and Spectral Data (5-7)}

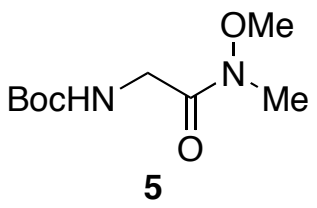

$N^{\alpha}$-(tert-Butoxycarbonyl)- $N$-methoxy- $N$-methyl-2-aminoacetamide (5). ${ }^{8}$ A modification of a reported procedure was utilized. ${ }^{9}$ To a $0{ }^{\circ} \mathrm{C}$ stirred solution of Boc-glycine (4) (21.0 g, 120. mmol) and $\mathrm{N}, \mathrm{O}$-dimethylhydroxylamine $(8.78 \mathrm{~g}, 144 \mathrm{mmol})$ (freshly prepared by treatment of $\mathrm{N}, \mathrm{O}$-dimethylhydroxylamine hydrochloride with triethanolamine in ethylene glycol followed by 
distillation $)^{10}$ in $\mathrm{CH}_{2} \mathrm{Cl}_{2}(400 \mathrm{~mL})$ was added DCC $(27.2 \mathrm{~g}, 132 \mathrm{mmol})$ and the reaction mixture was stirred at $0{ }^{\circ} \mathrm{C}$ for $2 \mathrm{~h}$ and then at $\mathrm{rt}$ for $20 \mathrm{~h}$. The reaction mixture was filtered through sintered glass to remove DCU, the filter cake was washed with $\mathrm{CH}_{2} \mathrm{Cl}_{2}(70 \mathrm{~mL})$, and the solvent was removed in vacuo to give a crude amorphous white solid. Recrystallization from ethyl acetate gave the desired product 5 as colorless needles in several crops (20.3 g, $93.1 \mathrm{mmol}, 78 \%$ yield): mp 102-103 ${ }^{\circ} \mathrm{C}\left(\right.$ lit. $\left.^{8 b} \mathrm{mp} 100-101{ }^{\circ} \mathrm{C}\right) ;{ }^{1} \mathrm{H} \mathrm{NMR}\left(\mathrm{CDCl}_{3}, 300 \mathrm{MHz}\right) \delta 5.22$ (br s, $\left.1 \mathrm{H}\right)$, $4.06(\mathrm{~d}, 2 \mathrm{H}, J=4.8 \mathrm{~Hz}), 3.69$ (s, $3 \mathrm{H}), 3.18(\mathrm{~s}, 3 \mathrm{H}), 1.43(\mathrm{~s}, 9 \mathrm{H}) \mathrm{ppm} ;{ }^{13} \mathrm{C} \mathrm{NMR}\left(\mathrm{CDCl}_{3}, 75\right.$ MHz) $\delta$ 170.4, 156.2, 79.9, 61.8, 42.0, 32.7, 28.6 ppm; Anal. calcd for $\mathrm{C}_{9} \mathrm{H}_{18} \mathrm{~N}_{2} \mathrm{O}_{4}: \mathrm{C}, 49.53 ; \mathrm{H}$, 8.31; N, 12.84. Found: C, 49.63; H, 8.40; N, 13.01.

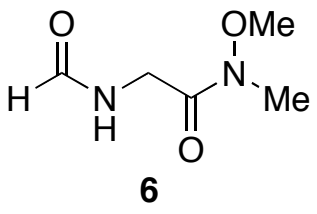

$N^{\alpha}$-Formyl- $\boldsymbol{N}$-methoxy- $\boldsymbol{N}$-methyl-2-aminoacetamide (6). ${ }^{9}$ Weinreb amide $5(25.1 \mathrm{~g}, 115$ mmol) and formic acid $(120 \mathrm{~mL})$ were combined, stirred, and heated to reflux for $1.5 \mathrm{~h}$. The solvent was removed in vacuo to give a crude tan solid (formate salt). The crude solid was treated with ethyl formate $(180 \mathrm{~mL})$ and triethylamine $(32.5 \mathrm{~mL}, 233 \mathrm{mmol})$ and the resulting mixture was heated to reflux for $16 \mathrm{~h}$. The solvent was removed in vacuo and the crude material was taken up in deionized water $(450 \mathrm{~mL})$ and extracted with $\mathrm{CH}_{2} \mathrm{Cl}_{2}(6 \times 400 \mathrm{~mL})$. The combined organic layers were dried over sodium sulfate. Removal of the solvent in vacuo gave the desired formamide 6 as a brown oil (10.9 g, $74.6 \mathrm{mmol}, 65 \%$ yield) which was utilized without further purification: ${ }^{1} \mathrm{H} \mathrm{NMR}\left(\mathrm{CDCl}_{3}, 300 \mathrm{MHz}\right) \delta 8.24(\mathrm{~s}, 1 \mathrm{H}), 6.51(\mathrm{br} \mathrm{s}, 1 \mathrm{H}), 4.21$ $(\mathrm{d}, 2 \mathrm{H}, J=4.5 \mathrm{~Hz}), 3.71(\mathrm{~s}, 3 \mathrm{H}), 3.20(\mathrm{~s}, 3 \mathrm{H}) \mathrm{ppm}$. 


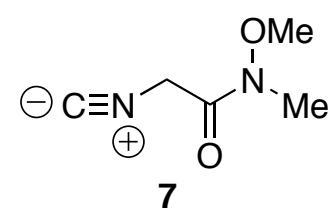

2-Isocyano- $N$-methoxy- $N$-methylacetamide (7) ${ }^{8 \mathrm{a}, 9}$ CAUTION: Manipulations involving isocyanide 7 should be performed in a hood (stench). A modification of a reported procedure was utilized. ${ }^{9}$ To a $0{ }^{\circ} \mathrm{C}$ stirred solution of formamide $\mathbf{6}(10.9,74.6 \mathrm{mmol})$ in THF $(240 \mathrm{~mL})$ was added triethylamine $(41.8 \mathrm{~mL}, 300$. mmol) followed by phosphorus oxychloride $(7.62 \mathrm{~mL}, 81.8$ mmol) dropwise via an addition funnel. The reaction mixture was stirred at $0{ }^{\circ} \mathrm{C}$ for $2 \mathrm{~h}$ and then at $\mathrm{rt}$ for $2 \mathrm{~h}$. The reaction mixture was then cooled to $0{ }^{\circ} \mathrm{C}$ and was treated with $\mathrm{CH}_{2} \mathrm{Cl}_{2}(300 \mathrm{~mL})$ followed by an aqueous solution of sodium bicarbonate $(300 \mathrm{~mL})$ dropwise via an addition funnel. This biphasic mixture was stirred for $1 \mathrm{~h}$ and then was treated with deionized water (400 $\mathrm{mL})$ and extracted with $\mathrm{CH}_{2} \mathrm{Cl}_{2}(8 \times 400 \mathrm{~mL})$. The combined organic layers were filtered through celite and the filter cake was washed with $\mathrm{CH}_{2} \mathrm{Cl}_{2}(2 \times 50 \mathrm{~mL})$. The organic solvent was removed in vacuo to give a crude orange/red solid that had a notably pungent isocyanide smell. Purification via flash chromatography (gradient: 1:3 to 1:2 ethyl acetate/petroleum ether) gave the desired product 7 as an off-white amorphous solid (6.73 g, $52.5 \mathrm{mmol}, 70 \%$ yield): $\mathrm{mp} 85-$ $86{ }^{\circ} \mathrm{C}$ (lit. $\left.{ }^{9} \mathrm{mp} 82^{\circ} \mathrm{C}\right) ; \mathrm{R}_{\mathrm{f}}=0.43\left(1: 1\right.$ ethyl acetate/petroleum ether); ${ }^{1} \mathrm{H} \mathrm{NMR}\left(\mathrm{CDCl}_{3}, 300 \mathrm{MHz}\right)$ $\delta 4.38$ (s, $2 \mathrm{H}), 3.70$ (s, $3 \mathrm{H}), 3.21$ (s, $3 \mathrm{H}) \mathrm{ppm} ;{ }^{13} \mathrm{C} \mathrm{NMR}\left(\mathrm{CDCl}_{3}, 75 \mathrm{MHz}\right) \delta 164.0,160.8,62.3$, 43.9, $32.6 \mathrm{ppm}$.

(iii) Spectral Data for Pyrrole Weinreb Amides 1a-1d

General Method: Synthesis of Pyrrole Weinreb Amides 1 from $\beta$-Nitroacetates 8. To a $0{ }^{\circ} \mathrm{C}$ stirred solution of isocyanide $7(0.51 \mathrm{~g}, 4.0 \mathrm{mmol})$ and $\mathrm{DBU}(2.1 \mathrm{~mL}, 14 \mathrm{mmol})$ in THF (10 
$\mathrm{mL})$ was added a solution of $\beta$-nitroacetate $8(5.6 \mathrm{mmol})$ in THF $(10 \mathrm{~mL})$ dropwise via syringe. The reaction mixture was stirred at $0{ }^{\circ} \mathrm{C}$ for $30 \mathrm{~min}$ and then at $\mathrm{rt}$ for $4 \mathrm{~h}$. The solvent was then removed in vacuo and the crude material obtained was purified by flash chromatography (ethyl acetate/petroleum ether gradient).

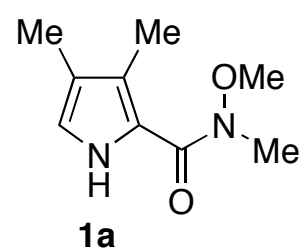

$N$-Methoxy- $N$-methyl-3,4-dimethylpyrrole-2-carboxamide (1a). The product was obtained as a light tan amorphous solid (89\% yield from 8a; 89\% yield from 9a). Recrystallization (95:5 hexane/ether) gave the analytical sample as a white crystals: mp 85-86 ${ }^{\circ} \mathrm{C} ; \mathrm{R}_{\mathrm{f}}=0.28$ (1:2 ethyl acetate/petroleum ether); IR (film) 3420, 2935, 1605, 1500, 1435, 1410, 1380, 1235, 1185, 1090, 975, 925, 865, $750 \mathrm{~cm}^{-1} ;{ }^{1} \mathrm{H} \mathrm{NMR}\left(\mathrm{CDCl}_{3}, 300 \mathrm{MHz}\right) \delta 9.20$ (br s, $\left.1 \mathrm{H}\right)$, $6.59(\mathrm{~d}, 1 \mathrm{H}, J=3.0 \mathrm{~Hz}), 3.68(\mathrm{~s}, 3 \mathrm{H}), 3.31(\mathrm{~s}, 3 \mathrm{H}), 2.29(\mathrm{~s}, 3 \mathrm{H}), 2.01(\mathrm{~s}, 3 \mathrm{H}) \mathrm{ppm} ;{ }^{13} \mathrm{C} \mathrm{NMR}$ $\left(\mathrm{CDCl}_{3}, 75 \mathrm{MHz}\right) \delta 162.2,128.3,120.1,120.0,119.0,61.5,33.6,11.0,10.1 \mathrm{ppm} ; \mathrm{MS} \mathrm{m} / \mathrm{z} 182$ $\left(\mathrm{M}^{+}\right), 152,136,122\left(\mathrm{M}^{+}-\mathrm{NMeOMe}\right), 94$; Anal. calcd for $\mathrm{C}_{9} \mathrm{H}_{14} \mathrm{~N}_{2} \mathrm{O}_{2}: \mathrm{C}, 59.32 ; \mathrm{H}, 7.74 ; \mathrm{N}$, 15.37. Found: C, 59.34; H, 7.88; N, 15.63.

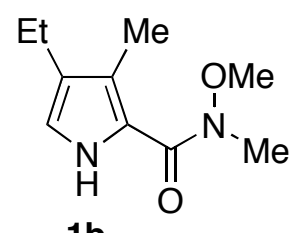

1b

4-Ethyl- $N$-methoxy- $N$-methyl-3-methylpyrrole-2-carboxamide (1b). The product was obtained as a yellow amorphous solid (95\% yield from $\mathbf{8 b} ; 92 \%$ yield from $\mathbf{9 b}$ ). 
Recrystallization (95:5 hexane/ether) gave the analytical sample as yellow crystals: mp $63-64{ }^{\circ} \mathrm{C}$; $R_{f}=0.40$ (1:2 ethyl acetate/petroleum ether); IR (film) 3380, 2960, 2930, 1600, 1510, 1410, 1230, 1180, 1090, 970, 920, 860, $750 \mathrm{~cm}^{-1} ;{ }^{1} \mathrm{H}$ NMR (CDCl $\left.3,300 \mathrm{MHz}\right) \delta 9.21$ (br s, $\left.1 \mathrm{H}\right), 6.59$ $(\mathrm{d}, 1 \mathrm{H}, J=2.7 \mathrm{~Hz}), 3.68(\mathrm{~s}, 3 \mathrm{H}), 3.29(\mathrm{~s}, 3 \mathrm{H}), 2.93(\mathrm{~s}, 3 \mathrm{H}), 2.42(\mathrm{q}, 2 \mathrm{H}, J=7.5 \mathrm{~Hz}), 1.15(\mathrm{t}, 3$ $\mathrm{H}, J=7.5 \mathrm{~Hz}) \mathrm{ppm} ;{ }^{13} \mathrm{C} \mathrm{NMR}\left(\mathrm{CDCl}_{3}, 75 \mathrm{MHz}\right) \delta 162.3,127.7,127.0,120.3,117.8,61.5,33.7$, 18.4, 14.7, 11.0 ppm; MS m/z $196\left(\mathrm{M}^{+}\right), 166,151,136\left(\mathrm{M}^{+}-\mathrm{NMeOMe}\right), 120,108$; Anal. calcd for $\mathrm{C}_{10} \mathrm{H}_{16} \mathrm{~N}_{2} \mathrm{O}_{2}$ : C, 61.20; H, 8.22; N, 14.27. Found: C, 61.12; H, 8.29; N, 14.27.

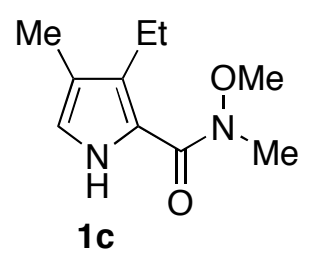

3-Ethyl- $N$-methoxy- $N$-methyl-4-methylpyrrole-2-carboxamide (1c). The product was obtained as a yellow/orange amorphous solid (85\% yield from 8c; $90 \%$ yield from 9c). Recrystallization (95:5 hexane/ether) gave the analytical sample as light yellow crystals: mp 73$74{ }^{\circ} \mathrm{C} ; \mathrm{R}_{\mathrm{f}}=0.45$ (1:2 ethyl acetate/petroleum ether); IR (film) 3300, 2975, 1585, 1555, 1495, $1410,1370,1235,1185,1080,1050,945,860,705 \mathrm{~cm}^{-1} ;{ }^{1} \mathrm{H}$ NMR $\left(\mathrm{CDCl}_{3}, 300 \mathrm{MHz}\right) \delta 9.24(\mathrm{br}$ s, $1 \mathrm{H}), 6.57(\mathrm{~d}, 1 \mathrm{H}, J=2.7 \mathrm{~Hz}), 3.67(\mathrm{~s}, 3 \mathrm{H}), 3.30(\mathrm{~s}, 3 \mathrm{H}), 2.79$ (q, $2 \mathrm{H}, J=7.4 \mathrm{~Hz}), 2.02(\mathrm{~s}, 3$ H), $1.12(\mathrm{t}, 3 \mathrm{H}, J=7.4 \mathrm{~Hz}) \mathrm{ppm} ;{ }^{13} \mathrm{C} \mathrm{NMR}\left(\mathrm{CDCl}_{3}, 75 \mathrm{MHz}\right) \delta 161.7,135.1,119.4,119.3$, 118.9, 61.4, 33.4, 18.6, 15.1, 9.8 ppm; MS m/z $196\left(\mathrm{M}^{+}\right), 166,136\left(\mathrm{M}^{+}\right.$- NMeOMe), 108; Anal. calcd for $\mathrm{C}_{10} \mathrm{H}_{16} \mathrm{~N}_{2} \mathrm{O}_{2}: \mathrm{C}, 61.20 ; \mathrm{H}, 8.22 ; \mathrm{N}, 14.27$. Found: $\mathrm{C}, 61.15 ; \mathrm{H}, 8.34 ; \mathrm{N}, 14.44$. 


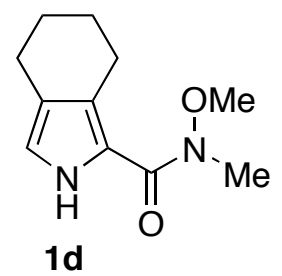

4,5,6,7-Tetrahydro- $N$-methoxy- $N$-methyl-2H-isoindole-1-carboxamide (1d). The product was obtained as a red amorphous solid (84\% yield from 9d). Recrystallization (10:1 hexane/ethyl acetate) gave the analytical sample as a reddish crystalline solid: $\mathrm{mp} 124-126{ }^{\circ} \mathrm{C}$; $\mathrm{R}_{\mathrm{f}}=0.36$ (1:2 ethyl acetate/petroleum ether); IR (film) 3490, 3215, 3030, 2995, 2970, 1615, $1570,1505,1450,1420,1355,1275,1100,970,925,900,870 \mathrm{~cm}^{-1} ;{ }^{1} \mathrm{H} \mathrm{NMR}\left(\mathrm{CDCl}_{3}, 300 \mathrm{MHz}\right)$

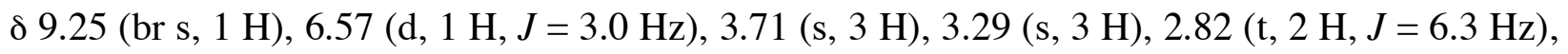
$2.54(\mathrm{t}, 2 \mathrm{H}, J=6.0 \mathrm{~Hz}), 1.69-1.74(\mathrm{~m}, 4 \mathrm{H}) \mathrm{ppm} ;{ }^{13} \mathrm{C} \mathrm{NMR}\left(\mathrm{CDCl}_{3}, 75 \mathrm{MHz}\right) \delta 161.8,130.6$, 121.7, 118.7, 117.6, 61.5, 33.5, 24.2, 23.8, 23.6, 22.2 ppm; MS m/z $209\left(\mathrm{M}^{+}+1\right), 208\left(\mathrm{M}^{+}\right), 178$, 177, 149, $148\left(\mathrm{M}^{+}-\mathrm{NMeOMe}\right), 121,120,119,118$; Anal. calcd for $\mathrm{C}_{11} \mathrm{H}_{16} \mathrm{~N}_{2} \mathrm{O}_{2}$ : C, 63.44; $\mathrm{H}$, 7.74; N, 13.45. Found: C, 63.23; H, 7.82; N, 13.52 .

(iv) Spectral Data for Pyrrole-2-carboxaldehydes 2a-2d

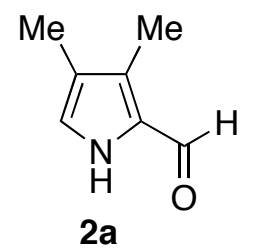

3,4-Dimethylpyrrole-2-carboxaldehyde (2a). ${ }^{11}$ The product was obtained as a beige amorphous solid (84\% yield): $\mathrm{mp} 129-130{ }^{\circ} \mathrm{C}$ (lit. $\left.{ }^{11 \mathrm{a}} \mathrm{mp} 133-134{ }^{\circ} \mathrm{C}\right) ; \mathrm{R}_{\mathrm{f}}=0.41$ (1:4 ethyl acetate/petroleum ether); ${ }^{1} \mathrm{H} \mathrm{NMR}\left(\mathrm{CDCl}_{3}, 300 \mathrm{MHz}\right) \delta 9.70$ (br s, $\left.1 \mathrm{H}\right), 9.54(\mathrm{~s}, 1 \mathrm{H}), 6.85(\mathrm{~d}, 1$ 
$\mathrm{H}, J=2.7 \mathrm{~Hz}), 2.45(\mathrm{~s}, 3 \mathrm{H}), 1.99(\mathrm{~s}, 3 \mathrm{H}) \mathrm{ppm} ;{ }^{13} \mathrm{C} \mathrm{NMR}\left(\mathrm{CDCl}_{3}, 75 \mathrm{MHz}\right) \delta 177.6,131.8$, 129.9, 125.5, 121.2, 9.7, 8.9 ppm; MS m/z $123\left(\mathrm{M}^{+}\right), 122,108,94$.

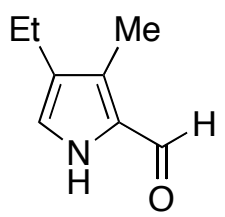

$2 b$

4-Ethyl-3-methylpyrrole-2-carboxaldehyde (2b). ${ }^{12}$ The product was obtained as a brown amorphous solid (69\% yield): $\mathrm{mp} 74-75{ }^{\circ} \mathrm{C}$ (lit. $\left.{ }^{12} \mathrm{mp} 77{ }^{\circ} \mathrm{C}\right) ; \mathrm{R}_{\mathrm{f}}=0.60$ (1:2 ethyl acetate/petroleum ether); ${ }^{1} \mathrm{H}$ NMR $\left(\mathrm{CDCl}_{3}, 300 \mathrm{MHz}\right) \delta 9.84$ (br s, $\left.1 \mathrm{H}\right), 9.56(\mathrm{~s}, 1 \mathrm{H}), 6.86(\mathrm{~d}, 1$ $\mathrm{H}, J=2.7 \mathrm{~Hz}), 2.41(\mathrm{q}, 2 \mathrm{H}, J=7.5 \mathrm{~Hz}), 2.26(\mathrm{~s}, 3 \mathrm{H}), 1.15(\mathrm{t}, 3 \mathrm{H}, J=7.5 \mathrm{~Hz}) \mathrm{ppm} ;{ }^{13} \mathrm{C} \mathrm{NMR}$ $\left(\mathrm{CDCl}_{3}, 75 \mathrm{MHz}\right) \delta 177.7,130.8,130.0,128.2,124.2,18.1,14.7,8.8 \mathrm{ppm} ; \mathrm{MS}$ m/z $137\left(\mathrm{M}^{+}\right)$, 136, 123, 122, 108, 94.

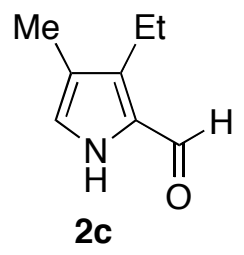

3-Ethyl-4-methylpyrrole-2-carboxaldehyde (2c). ${ }^{13}$ The product was obtained as a brown amorphous solid $\left(65 \%\right.$ yield): $\mathrm{mp} 74-75{ }^{\circ} \mathrm{C}$ (lit. $\left.{ }^{13} \mathrm{mp} 74.5-76{ }^{\circ} \mathrm{C}\right) ; \mathrm{R}_{\mathrm{f}}=0.68$ (1:2 ethyl acetate/petroleum ether); ${ }^{1} \mathrm{H}$ NMR $\left(\mathrm{CDCl}_{3}, 300 \mathrm{MHz}\right) \delta 9.57$ (s, $\left.1 \mathrm{H}\right), 8.83(\mathrm{br} \mathrm{s}, 1 \mathrm{H}), 6.80$ (d, 1 $\mathrm{H}, J=3.0 \mathrm{~Hz}), 2.71(\mathrm{q}, 2 \mathrm{H}, J=7.5 \mathrm{~Hz}), 2.03(\mathrm{~s}, 3 \mathrm{H}), 1.19(\mathrm{t}, 3 \mathrm{H}, J=7.5 \mathrm{~Hz}) \mathrm{ppm} ;{ }^{13} \mathrm{C} \mathrm{NMR}$ $\left(\mathrm{CDCl}_{3}, 75 \mathrm{MHz}\right) \delta 177.6,138.3,129.2,125.7,120.5,17.3,16.8,9.7 \mathrm{ppm} ; \mathrm{MS} \mathrm{m} / \mathrm{z} 137\left(\mathrm{M}^{+}\right)$, $136,122,108,94$. 


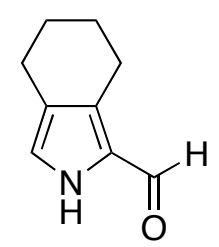

2d

4,5,6,7-Tetrahydro- $2 \mathrm{H}$-isoindole-1-carboxaldehyde $(\mathbf{2 d}) .{ }^{14}$ The product was obtained as a red amorphous solid (59\% yield): $\mathrm{mp} 116-117{ }^{\circ} \mathrm{C}\left(\right.$ lit. $\left.^{14} \mathrm{mp} 117.5-118{ }^{\circ} \mathrm{C}\right) ; \mathrm{R}_{\mathrm{f}}=0.45(1: 1$ ethyl acetate/petroleum ether); ${ }^{1} \mathrm{H}$ NMR (d ${ }_{6}$-DMSO, $\left.300 \mathrm{MHz}\right) \delta 12.42$ (br s, $\left.1 \mathrm{H}\right), 10.28$ (s, $\left.1 \mathrm{H}\right), 7.71$ $(\mathrm{d}, 1 \mathrm{H}), 3.55-3.61(\mathrm{~m}, 4 \mathrm{H}), 3.26-3.33(\mathrm{~m}, 4 \mathrm{H}) \mathrm{ppm} ;{ }^{13} \mathrm{C}$ NMR $\left(\mathrm{d}_{6}-\mathrm{DMSO}, 75 \mathrm{MHz}\right) \delta 176.8$, 127.6, 123.4, 123.2, 121.3, 23.0, 22.6, 21.1, 20.9 ppm; MS (m/z) $149\left(\mathrm{M}^{+}\right), 148,132,121,120$.

(v) Spectral Data for 3-Pyrrolin-2-ones 3a-3d (1H-Pyrrol-2(5H)-ones)

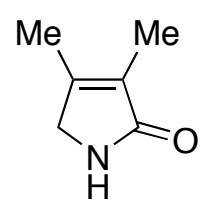

$3 a$

3,4-Dimethyl-3-1H-pyrrol-2(5H)-one (3a). ${ }^{1,15}$ The product was obtained as a white amorphous solid (50\% yield): $\mathrm{mp} 119-121{ }^{\circ} \mathrm{C}$ (lit. $\left.{ }^{1} \mathrm{mp} 114-116{ }^{\circ} \mathrm{C}\right) ; \mathrm{R}_{\mathrm{f}}=0.16$ (4:1 ethyl acetate/petroleum ether); ${ }^{1} \mathrm{H}$ NMR $\left(\mathrm{CDCl}_{3}, 300 \mathrm{MHz}\right) \delta 7.64$ (br s, $\left.1 \mathrm{H}\right), 3.76(\mathrm{~s}, 2 \mathrm{H}), 1.92$ (s, 3 $\mathrm{H}), 1.74$ (s, $3 \mathrm{H}) \mathrm{ppm} ;{ }^{13} \mathrm{C} \mathrm{NMR}\left(\mathrm{CDCl}_{3}, 75 \mathrm{MHz}\right) \delta 176.9,149.2,128.6,50.0,13.4,8.4$ ppm; MS m/z $111\left(\mathrm{M}^{+}\right), 96,82$.

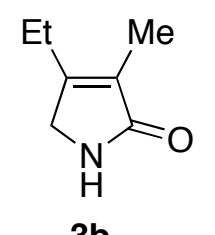


4-Ethyl-3-methyl-1H-pyrrol-2(5H)-one $(\mathbf{3 b}){ }^{16}$ The product was obtained as a white amorphous solid (73\% yield): $\mathrm{mp} 78-79{ }^{\circ} \mathrm{C}$ (lit. $\left.{ }^{16 \mathrm{a}} \mathrm{mp} 78-80{ }^{\circ} \mathrm{C}\right) ; \mathrm{R}_{\mathrm{f}}=0.21$ (4:1 ethyl acetate/petroleum ether); ${ }^{1} \mathrm{H} \mathrm{NMR}\left(\mathrm{CDCl}_{3}, 300 \mathrm{MHz}\right) \delta 7.92$ (br s, $\left.1 \mathrm{H}\right), 3.76(\mathrm{~d}, 2 \mathrm{H}, \mathrm{J}=0.6 \mathrm{~Hz})$, $2.33(\mathrm{q}, 2 \mathrm{H}, J=7.5 \mathrm{~Hz}), 1.72(\mathrm{~m}, 3 \mathrm{H}), 1.03(\mathrm{t}, 3 \mathrm{H}, J=7.5 \mathrm{~Hz}) \mathrm{ppm} ;{ }^{13} \mathrm{C} \mathrm{NMR}\left(\mathrm{CDCl}_{3}, 75\right.$ MHz) $\delta 177.0,154.9,127.7,48.3,21.2,12.9,8.3$ ppm; MS m/z 126, $125\left(\mathrm{M}^{+}\right), 110,96$.

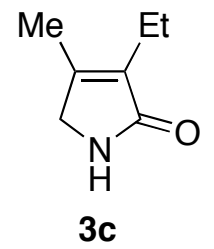

3-Ethyl-4-methyl-1H-pyrrol-2(5H)-one $(\mathbf{3 c}){ }^{16 b, 17}$ The product was obtained as a white amorphous solid (67\% yield): $\mathrm{mp} 100-102{ }^{\circ} \mathrm{C}$ (lit. ${ }^{17} \mathrm{mp} \mathrm{101-102}{ }^{\circ} \mathrm{C}$ ); $\mathrm{R}_{\mathrm{f}}=0.26$ (4:1 ethyl acetate/petroleum ether); ${ }^{1} \mathrm{H}$ NMR $\left(\mathrm{CDCl}_{3}, 300 \mathrm{MHz}\right) \delta 7.78$ (br s, $\left.1 \mathrm{H}\right), 3.75$ (s, $\left.2 \mathrm{H}\right), 2.22$ (q, 2 $\mathrm{H}, J=7.5 \mathrm{~Hz}), 1.94(\mathrm{~s}, 3 \mathrm{H}), 1.12(\mathrm{t}, 3 \mathrm{H}, J=7.5 \mathrm{~Hz}) \mathrm{ppm} ;{ }^{13} \mathrm{C} \mathrm{NMR}\left(\mathrm{CDCl}_{3}, 75 \mathrm{MHz}\right) \delta 176.5$, 148.9, 134.2, 50.3, 16.8, 13.3, 13.2 ppm; MS m/z 126, $125\left(\mathrm{M}^{+}\right), 110,96$.

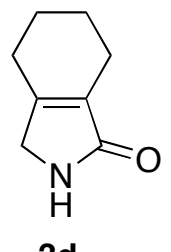

3d

2,3,4,5,6,7-Hexahydroisoindol-1-one $(\mathbf{3 d}){ }^{18}$ The product was obtained as a white amorphous solid (56\% yield): $\mathrm{mp} 138-140{ }^{\circ} \mathrm{C}$ (lit. ${ }^{18 \mathrm{a}} \mathrm{mp} 115-116{ }^{\circ} \mathrm{C}$ ); $\mathrm{R}_{\mathrm{f}}=0.16$ (4:1 ethyl acetate/petroleum ether); ${ }^{1} \mathrm{H} \mathrm{NMR}\left(\mathrm{CDCl}_{3}, 300 \mathrm{MHz}\right) \delta 7.56$ (br s, $\left.1 \mathrm{H}\right), 3.78(\mathrm{~d}, 2 \mathrm{H}), 2.15-2.23$ (m, $4 \mathrm{H}), 1.66-1.75(\mathrm{~m}, 4 \mathrm{H}) \mathrm{ppm} ;{ }^{13} \mathrm{C} \mathrm{NMR}\left(\mathrm{CDCl}_{3}, 75 \mathrm{MHz}\right) \delta 176.0,153.5,131.6,49.3,24.6$, 
22.2, 22.0, $20.1 \mathrm{ppm}$; MS m/z $137\left(\mathrm{M}^{+}\right), 122,108,95$. (note: although $\mathrm{mp}$ is off, ${ }^{13} \mathrm{C} \mathrm{NMR}$ is consistent with reference 18a)

\section{(vi) References}

(1) Chen, Q.; Wang, T.; Zhang, Y.; Wang, Q.; Ma, J. Synth. Commun. 2002, 32, 1031-1040.

(2) Barton, D.H.R.; Kervagoret, J.; Zard, S.Z. Tetrahedron 1990, 46, 7587-7598.

(3) Ballini, R.; Castagnani, R.; Petrini, M. J. Org. Chem. 1992, 57, 2160-2162.

(4) Houssin, R.; Bernier, J.-L.; Hénichart, J.-P. Synthesis 1988, 259-261.

(5) Corey, E.J.; Estreicher, H. J. Am. Chem. Soc. 1978, 100, 6294-6295.

(6) Robertson, D.N. J. Org. Chem. 1960, 25, 47-50.

(7) (a) Kornblum, N.; Larson, H.O.; Blackwood, R.K.; Mooberry, D.D.; Oliveto, E.P.; Graham, G.E. J. Am. Chem. Soc. 1956, 78, 1497-1501; (b) Ballini, R.; Barboni, L.; Giarlo, G. J. Org. Chem. 2004, 69, 6907-6908.

(8) (a) Kim, S.W.; Bauer, S.M.; Armstrong, R.W. Tetrahedron Lett. 1998, 39, 6993-6996; (b) Brown, P.; Davies, D.T.; O’Hanlon, P.J.; Wilson, J.M. J. Med. Chem. 1996, 39, 446-457; (c) Löser, R.; Schilling, K.; Dimming, E.; Gütschow, M. J. Med. Chem. 2005, 48, 76887707.

(9) Sawamura, M.; Nakayama, Y.; Kato, T.; Ito, Y. J. Org. Chem. 1995, 60, 1727-1732.

(10) Hung, D.T.; Nerenberg, J.B.; Schreiber, S.L. J. Am. Chem. Soc. 1996, 118, 11054-11080.

(11) (a) Hwang, K.-O.; Lightner, D.A. Tetrahedron 1994, 50, 1955-1966; (b) de Groot, J.A.; Gorter-La Roy, G.M.; van Koeveringe, J.A.; Lugtenburg, J. Org. Prep. Proc. Int. 1981, $13,97-101$. 
(12) Smith, K.M.; Bobe, F.W.; Minnetian, O.M.; Hope, H.; Yanuck, M.D. J. Org. Chem. 1985, 50, 790-792.

(13) Paine, III, John B.; Dolphin, D. J. Org. Chem. 1988, 53, 2787-2795.

(14) Lash, T.D. J. Porphyrins Phthalocyanines 1997, 1, 29-44.

(15) (a) Xie, M.; Lightner, D.A. Tetrahedron 1993, 49, 2185-2200; (b) Byun, Y.-S. ; Lightner, D.A. J. Org. Chem. 1991, 56, 6027-6033.

(16) (a) Chen, Q.; Huggins, M.T.; Lightner, D.A.; Norona, W.; McDonagh, A.F. J. Am. Chem. Soc. 1999, 121, 9253-9264; (b) Schoenleber, R.W.; Kim, Y.; Rapoport, H. J. Am. Chem. Soc. 1984, 106, 2645-2651.

(17) Bishop, J.E.; Nagy, J.O.; O'Connell, J.F.; Rapoport, H. J. Am. Chem. Soc. 1991, 113, 8024-8025.

(18) (a) Crisp, G.T.; Meyer, A.G. Tetrahedron 1995, 51, 5585-5596; (b) Armarego, W.L.F.; Kobayashi, T. J. Chem. Soc. (C) 1971, 3222-3229. 